\title{
A RIGOROUS JUSTIFICATION OF THE REYNOLDS EQUATION*
}

\author{
BY \\ GIOVANNI CIMATTI \\ University of Pisa, Pisa, Italy
}

\begin{abstract}
A small parameter technique is used to derive Reynolds' lubrication equation from the Stokes equation. The error associated with the approximation is estimated in suitable norms.
\end{abstract}

1. Introduction. In a classical paper [9], O. Reynolds formulated a differential equation which gives an asymptotic approximation of the Navier-Stokes equation. In the one-dimensional case this equation reads as follows:

$$
\left(H^{3} P^{\prime}\right)^{\prime}=6 \mu \bar{V} H^{\prime} .
$$

$P$ is the pressure in a thin film of a viscous fluid of constant density, thickness $H$ and viscosity $\mu . \bar{V}$ is the $X_{1}$-component of the moving surface. Equation (1.1) applies when the height of the fluid is very small compared to the span and the length. The justifications given in the literature for the transition from the full Navier-Stokes equations to (1.1) are mainly heuristic; we quote in particular the papers of Elrod [3] and Wannier [10].

A possible approach to the Reynolds equation, and thus to the theory of lubrication, is to suppose true the expressions of the Couette-Poiseuille flow,

$$
V_{1}=\frac{1}{2 \mu} \frac{\partial P}{\partial X_{1}} X_{2}\left(X_{2}-H\right)+\bar{V}\left(H-X_{2}\right) / H,
$$

even when $H$ is not a constant. In the first part of this paper (Secs. 2 and 3) we examine in detail the asymptotic validity of this assumption of the theory.

In Sec. 4 we develop a formal expansion which gives in the first term the Reynolds equation. Various rigorous estimates of the remainder are proved. In this paper we limit ourselves to the two-dimensional linear case (i.e., we consider as "exact" the solution given by the linear Stokes equations).

The transition from the Stokes equations to the Reynolds equation in the three-dimensional case (but only to the first order) is studied in a paper by G. Bayada and M.

* Received January 3, 1986. 
Chambat [11], which recently appeared and which we saw after completing the present work. No use is made in [11] of the technique of the stream function on which this paper is largely based.

2. Generalized Couette-Poiseuille flow. Let $H\left(X_{1}\right) \in C^{\infty}\left(R^{1}\right)$ be a periodic function of period $L$ such that $H_{M} \geqslant H \geqslant H_{m}>0$. Define

$$
\begin{aligned}
& S=\left\{\left(X_{1}, X_{2}\right) ;-\infty<X_{1}<\infty, 0<X_{2}<H\left(X_{2}\right)\right\}, \\
& \Omega=\left\{\left(X_{1}, X_{2}\right) ; 0<X_{1}<L, 0<X_{2}<H\left(X_{1}\right)\right\} .
\end{aligned}
$$

First of all we study the following.

Problem 1. To find $\mathbf{V}=\left(V_{1}, V_{2}\right)$ such that

$$
\begin{gathered}
\mu \Delta \mathbf{V}=\nabla P \text { in } S, \\
\nabla \cdot \mathbf{V}=0, \\
V_{1}=\bar{V}, V_{2}=0 \text { on } X_{2}=0, \quad \mathbf{V}=0 \text { on } X_{2}=H\left(X_{1}\right), \\
\mathbf{V}\left(X_{1}+L, X_{2}\right)=\mathbf{V}\left(X_{1}, X_{2}\right), \quad L>0 \text { a given constant. }
\end{gathered}
$$

$\mathbf{V}$ is the velocity of the plane flow we discuss, $P$ the pressure, $\mu$ the (constant) viscosity and $\bar{V}$ a given constant. If $H=H_{m}$ the solution of Problem 1 is of course the well-known Couette-Poiseuille formula

$$
\mathbf{V}=\mathbf{V}^{(C)}+\mathbf{V}^{(P)}
$$

where

$$
\begin{aligned}
V_{1}^{(C)}=\frac{K}{2 \mu L} X_{2}\left(X_{2}-H_{m}\right), \quad V_{1}^{(P)} & =\bar{V}\left(1-X_{2} / H_{m}\right), \quad V_{2}^{(C)}=0, \quad V_{2}^{(P)}=0 . \\
P & =\frac{K}{L} X_{1}
\end{aligned}
$$

with $K$ an arbitrary constant. Hence in this elementary case (a) the solution of Problem 1 is unique when $K$ is assigned or if

$$
C=\int_{0}^{H_{m}} V_{1}\left(X_{2}\right) d X_{2}
$$

is prescribed. The one-to-one correspondence between $C$ and $K$ is easily computed:

$$
C=\frac{-K H_{m}^{3}}{12 L \mu}+\frac{H_{m} \bar{V}}{2}
$$

Moreover (b) the pressure is periodic only if $K=0$ and in this case $\mathbf{V}=\mathbf{V}^{(P)}$. We are going to show that properties (a) and (b) remain true when $H$ is an arbitrary periodic function. If $\mathbf{V}$ is a regular solution of Problem 1 then $P_{X_{1}}$ is periodic with respect to $X_{1}$; thus

$$
P\left(X_{1}, X_{2}\right)=G\left(X_{1}, X_{2}\right)+A\left(X_{2}\right) X_{1}+B\left(X_{2}\right)
$$


where $G\left(X_{1}, X_{2}\right)$ is $L$-periodic in $X_{1}$. Hence

$$
P\left(X_{1}+L, X_{2}\right)-P\left(X_{1}, X_{2}\right)=A\left(X_{2}\right) L .
$$

But $P_{X_{2}}$ is also periodic in $X_{1}$; thus $A$ is constant. Defining $K=A L$ we have

$$
P\left(X_{1}+L, X_{2}\right)-P\left(X_{1}, X_{2}\right)=K \text {. }
$$

We give now a weak formulation of Problem 1 in which the constant $K$ appears explicitly following the standard functional approach (see e.g. [6]).

Let $\dot{J}(S)$ be the set of all sufficiently smooth solenoidal two-dimensional vectors vanishing near $\partial S$ which are $L$-periodic in $X_{1}$. In $\dot{J}(S)$ we introduce the scalar product ${ }^{1}$

and the norm

$$
[\mathbf{U}, \mathbf{V}]=\int_{\Omega} \mathbf{U}_{X_{k}} \cdot \mathbf{V}_{\mathbf{X}_{k}} d X, \quad d X=d X_{1} d X_{2}
$$

$$
\|\mathbf{U}\|=[\mathbf{U}, \mathbf{U}]^{1 / 2}
$$

Denote by $H(S)$ the completion of $\dot{J}(S)$ with respect to (2.9). Multiplying (2.1) by $\Phi \in H$ and integrating by parts over $\Omega$ we have, recalling (2.8),

$$
-\mu[\mathbf{V}, \boldsymbol{\Phi}]=K \int_{0}^{H(0)} \Phi_{1}\left(0, X_{2}\right) d X_{2},
$$

for all $\Phi \in H$. The integral on the right-hand side of (2.10) makes sense since $\Phi_{1} \in L^{2}(\partial \Omega)$. Let $\mathbf{A}$ be any smooth solenoidal vector field which satisfies (2.3) and (2.4). If we define $\mathbf{U}=\mathbf{V}-\mathbf{A}$ we can write (2.10) in the form

$$
\mathbf{U} \in H, \quad-\mu[\mathbf{U}, \boldsymbol{\Phi}]=K \int_{0}^{H(0)} \Phi_{1}\left(0, X_{2}\right) d X_{2}-[\mathbf{A}, \boldsymbol{\Phi}] \mu .
$$

The right-hand side in (2.11) defines a linear functional in $H(S)$. Thus by the Riesz representation theorem we have

TheOREM 2.1. For every $K \in \mathbf{R}^{1}$ there exists one and only one solution of Problem (2.10).

The regularity of the solution together with other properties, is better obtained using a stream function $\Psi\left(X_{1}, X_{2}\right)$ defined by

$$
\Psi_{X_{2}}=V_{1}, \quad \Psi_{X_{1}}=-V_{2} .
$$

In terms of $\Psi$, Problem 1 becomes

Problem 2. To find $\Psi$ such that

$$
\begin{gathered}
\Delta^{2} \Psi=0 \quad \text { in } S, \\
\Psi=0, \quad \Psi_{X_{2}}=\bar{V} \quad \text { on } \quad X_{2}=0, \\
\Psi=C, \quad \frac{d \Psi}{d n}=0 \quad \text { on } \quad X_{2}=H\left(X_{1}\right), \\
\Psi\left(X_{1}+L, X_{2}\right)=\Psi\left(X_{1}, X_{2}\right) .
\end{gathered}
$$

$d / d n$ is the normal derivative along $\partial S$. We note that the stream function is constant on $\partial S$ by the boundary conditions imposed on $\mathbf{V}$. Thus we can assume $\Psi=0$ on $X_{2}=0$; however, since $\partial S$ consists of two disjunct arcs, we have $\Psi=C$ on $X_{2}=H\left(X_{1}\right)$ and in

${ }^{1}$ Use is made of the summation convention. 
general $C \neq 0$. The physical meaning of $C$ is readily seen. Indeed

$$
C=\int_{0}^{H(0)} \Psi_{X_{2}}\left(0, X_{2}\right) d X_{2}=\int_{0}^{H(0)} V_{1}\left(0, X_{2}\right) d X_{2} .
$$

With $C$ prescribed, the theory of linear boundary value problems applies to (2.13)-(2.16) with the minor changes required by the condition of periodicity. Hence the solution of Problem 2 exists, is unique and of class $C^{\infty}(\bar{S})$ since we suppose $H \in C^{\infty}(\bar{S})$ and $\partial S \in C^{\infty}$. In the following theorem we show that the formulations of Problems 1 and 2 are perfectly equivalent. This, in particular, proves the $C^{\infty}$ regularity of the solution of Problem 1.

THEOREM 2.2. The correspondence $C=F(K)$ is one-to-one from $\mathbf{R}^{1}$ on $\mathbf{R}^{1}$.

Proof. Let $K \in \mathbf{R}^{1}$. By Theorem 2.1 there exists one and only one solution of Problem 1; thus

$$
C=F(K)=\int_{0}^{H(0)} V_{1}\left(0, X_{2}\right) d X_{2}
$$

is well-defined. We say that $F$ is one-to-one. Let $\mathbf{V}^{1}$ and $\mathbf{V}^{2}$ be the solutions of Problem 1 corresponding to $K_{1}$ and $K_{2}$ with $K_{1} \neq K_{2}$. Suppose by contradiction $C_{1}=C_{2}$ and define $\mathbf{W}=\mathbf{V}^{1}-\mathbf{V}^{2}$. From (2.10) we have

$$
-\mu[\mathbf{W}, \Phi]=\left(K_{1}-K_{2}\right) \int_{0}^{H(0)} \Phi_{1}\left(0, X_{2}\right) d X_{2} .
$$

With $\Phi=\mathbf{W}$ we get

$$
-\mu\|\mathbf{W}\|_{H}^{2}=\left(K_{1}-K_{2}\right)\left(C_{1}-C_{2}\right)=0
$$

and this cannot be since $\|\mathbf{W}\|_{H} \neq 0$.

To prove that $F\left(\mathbf{R}^{1}\right)=\mathbf{R}^{1}$ we pick $\bar{C} \in \mathbf{R}^{1}$ and let $\Psi$ be the corresponding unique solution of Problem 2. Now $\mathbf{V}=\left(\Psi_{X_{2}},-\Psi_{X_{1}}\right)$ is a solution to Problem 1. Let $P$ be related pressure. Since $\nabla P$ is periodic in $X_{1}$ there exists a constant $\bar{K}$ such that

$$
P\left(X_{1}+L, X_{2}\right)-P\left(X_{1}, X_{2}\right)=\bar{K}
$$

and clearly $\bar{C}=F(\bar{K})$.

In analogy with the case $H=H_{m}$ we call $\mathbf{V}^{(P)}$ the solution to Problem 1 when $K=0$. If $K$ is arbitrary and $\mathbf{V}$ the corresponding solution we put

$$
\mathbf{V}^{(C)}=\mathbf{V}-\mathbf{V}^{(P)} \text {. }
$$

THEOREM 2.3. The pressure is periodic with respect to $X_{1}$ (i.e., $K=0$ ) if and only if

$$
\int_{0}^{H(0)} V_{1}\left(0, X_{2}\right) d X_{2}=\int_{0}^{H(0)} V_{1}^{(P)}\left(0, X_{2}\right) d X_{2} .
$$

Proof. If $K=0$ then (2.17) follows by definition. Vice versa suppose (2.17) holds. We have

$$
\int_{0}^{H(0)} V_{1}^{(C)}\left(0, X_{2}\right) d X_{2}=0
$$

Now $\mathbf{V}^{(C)}$ satisfies

$$
\Delta \mathbf{V}^{(C)}=\nabla P^{(C)}, \quad \nabla \cdot \mathbf{V}^{(C)}=0 \quad \text { in } S,
$$




$$
\mathbf{V}^{(C)}=0 \quad \text { on } \quad \partial S, \quad \mathbf{V}^{(C)}\left(X_{1}+L, X_{2}\right)=\mathbf{V}^{(C)}\left(X_{1}, X_{2}\right) \quad \text { in } \quad S .
$$

Multiplying $(2.19)_{1}$ by $\mathbf{V}^{(C)}$ we obtain from $(2.19)_{2}$ and (2.18)

$$
\left\|\mathbf{V}^{(C)}\right\|_{H}=0
$$

Hence $\mathbf{V}^{(C)}=0$. This implies $K=0$.

3. Asymptotic properties of the generalized Couette-Poiseuille flow. In this section we reformulate Problems 1 and 2 in the variable domain:

$$
S_{\varepsilon}=\left\{\left(X_{1}, X_{2}\right) \in \mathbf{R}^{2},\left|X_{1}\right|<\infty, 0<X_{2}<\varepsilon H\left(X_{1}\right)\right\}
$$

and then let $\varepsilon \rightarrow 0$. Since $\varepsilon$ is a small parameter we prefer to work with nondimensional variables. Thus we define

$$
\begin{gathered}
x=\left(x_{1}, x_{2}\right), x_{1}=X_{1} / L, x_{2}=X_{2} / H_{m}, h\left(x_{1}\right)=H\left(L x_{1}\right) / H_{m}, \mathbf{v}=\mathbf{V} / \bar{V}, \mathbf{v}=\left(v_{1}, v_{2}\right), \\
p=\frac{P L}{\mu \bar{V}}, k=\frac{K H_{m}^{2}}{\mu \bar{V} L}, c=\frac{C}{\bar{V} H_{m}}, \psi=\frac{\Psi}{\bar{V} H_{m}}, h_{m} \geqslant h\left(x_{1}\right) \geqslant h_{m}, \\
s_{\varepsilon}=\left\{\left(x_{1}, x_{2}\right) ;\left|x_{1}\right|<\infty, 0<x_{2}<\varepsilon h\left(x_{1}\right)\right\}, \\
o_{\varepsilon}=\left\{\left(x_{1}, x_{2}\right) ; 0<x_{1}<1,0<x_{2}<\varepsilon h\left(x_{1}\right)\right\},
\end{gathered}
$$

$\varepsilon$ a nondimensional parameter. Problems 1 and 2 become

Problem 1'. To find $\mathbf{v}$ such that

$$
\begin{gathered}
\Delta \mathbf{v}=\nabla p, \quad \nabla \cdot \mathbf{v}=0 \text { in } s_{\varepsilon}, \\
v_{1}=1, v_{2}=0 \quad \text { on } \quad x_{2}=0, \quad \mathbf{v}=0 \quad \text { on } \quad x_{2}=\varepsilon h\left(x_{1}\right), \\
\mathbf{v}\left(x_{1}+1, x_{2}\right)=\mathbf{v}\left(x_{1}, x_{2}\right) .
\end{gathered}
$$

Problem 2'. To find $\psi$ such that

$$
\begin{gathered}
\Delta^{2} \psi=0 \quad \text { in } \quad s_{\varepsilon}, \\
\psi=0, \psi_{x_{2}}=1 \quad \text { on } \quad x_{2}=0, \quad \psi=c, \frac{d \psi}{d n}=0 \quad \text { on } \quad x_{2}=\varepsilon h\left(x_{1}\right), \\
\psi\left(x_{1}+1, x_{2}\right)=\psi\left(x_{1}, x_{2}\right) .
\end{gathered}
$$

The Couette-Poiseuille solution corresponding to $h\left(x_{1}\right)=1$ is, in this notation,

$$
v_{1}\left(x_{2}\right)=v_{1}^{(P)}+v_{1}^{(C)}, \quad v_{2}=0
$$

where

$$
v_{1}^{(C)}=\frac{k}{2} x_{2}\left(x_{2}-\varepsilon\right), \quad v_{1}^{(P)}=\left(\varepsilon-x_{2}\right) / \varepsilon .
$$

We are interested in solutions of Problems $1^{\prime}$ for which $v_{1}^{(C)}$ does not vanish and does not diverge as $\varepsilon \rightarrow 0$. Of course this is equivalent to saying that $k=k_{0} / \varepsilon^{2}$. This is the case in the theory of lubrication and hereafter we will always make this assumption. We easily 
verify, when $k=k_{0} / \varepsilon^{2}$ in (3.7),

$$
\begin{gathered}
\left\|v_{1}\right\|_{L^{1}\left(o_{f}\right)}=C_{1} \varepsilon, \\
\left\|v_{1}\right\|_{L^{p}\left(o_{r}\right)}=C_{p} \varepsilon^{1 / p}, \quad 1<p<\infty, \\
\left\|v_{1}\right\|_{L^{\infty}\left(o_{\varepsilon}\right)}=C_{\infty}, \\
\left\|\nabla v_{1}\right\|_{L^{2}\left(o_{\varepsilon}\right)}=\tilde{C} \varepsilon^{-1 / 2} .
\end{gathered}
$$

Moreover, if $k=k_{0} / \varepsilon^{2}$ in (3.7), then

$$
c=c_{0} \varepsilon, \quad c_{0}=-k_{0} / 12+1 / 2 .
$$

In terms of $c_{0},(3.7)$ becomes

$$
v_{1}\left(x_{2}\right)=3\left(1-2 c_{0}\right) x_{2}^{2} / \varepsilon^{2}+2\left(3 c_{0}-2\right) x_{2} / \varepsilon+1 .
$$

The basic idea of the theory of lubrication is to consider (3.13) still valid when $h$ depends on $x_{1}$ and $\varepsilon$ is very small. To make this point precise we prove that the estimates (3.8)-(3.12) remain valid for the generalized Couette-Poiseuille flow and that the exact solution of Problem $1^{\prime}$ converges in suitable norms to the flow of the theory of lubrication.

Let $H_{p 0}^{1}\left(s_{\varepsilon}\right)$ be the completion with respect to the norm

$$
\|u\|_{H_{p 0}^{1}}=\left(\int_{O_{e}}|\nabla u|^{2} d x\right)^{1 / 2}, \quad d x=d x_{1} d x_{2}
$$

of the $C^{\infty}$-functions vanishing near $\partial s_{\varepsilon}$ and 1-periodic in $x_{1}$. For the proof of the following Poincaré-like lemma compare also [6], p. 8.

Lemma 3.1. Suppose $u \in H_{p 0}^{1}$. We have

$$
\|u\|_{L^{2 p}\left(o_{r}\right)} \leqslant C(p) \varepsilon^{1 / p}\|\nabla u\|_{L^{2}\left(o_{e}\right)}
$$

where

$$
C(p)=\left(h_{M}(p !)^{2} / 2^{p-1}\right)^{1 / p}, \quad 1 \leqslant p<\infty .
$$

Proof. It suffices to prove (3.14) when $u \in C^{\infty}\left(s_{\varepsilon}\right)$ vanishes near $\partial s_{\varepsilon}$ and is 1-periodic in $x_{1}$. For $p=1$ (3.14) certainly holds. Now

$$
\begin{aligned}
\int_{O_{e}} u^{2(p+1)} d x & \leqslant(p+1)^{2} \int_{o_{e}}\left|u^{p} u_{x_{1}}\right| d x \int_{O_{\epsilon}}\left|u^{p} u_{x_{2}}\right| d x \\
& \leqslant \frac{1}{2}(p+1)^{2}\|u\|_{L^{2 p}}^{2}\|\nabla u\|_{L^{2} .}^{2}
\end{aligned}
$$

Suppose (3.14). Then by (3.15)

$$
\|u\|_{L^{2(p+1)}} \leqslant\left(\varepsilon h_{M}(p+1) ! / 2^{p}\right)^{1 /(p+1)}\|\nabla u\|_{L^{2}} .
$$

Hence by induction the result follows.

In the following three lemmas we recall various "a priori" estimates for the solutions of harmonic and biharmonic problems. 
LEMMA 3.2. (C. Miranda [7]) Let $\Omega$ be an open, bounded, and connected subset of $\mathbf{R}^{2}$ with a regular boundary (e.g. $\partial \Omega \in C^{2}$ ). Suppose $v \in C^{1}(\bar{\Omega}) \cap C^{4}(\Omega)$ and

$$
\Delta^{2} v=0 \text { in } \Omega, \quad v=0 \text { on } \partial \Omega .
$$

Then

$$
|\nabla v| \leqslant(56)^{1 / 2} \max _{\partial \Omega}|d v / d n| .
$$

Take $\Omega$ as in Lemma 3.2. Let $B_{x}\left(r_{i}\right),\left(B_{x}\left(r_{e}\right)\right)$ be the greatest open disk of radius $r_{i}\left(r_{e}\right)$ internally (externally) tangent to $\partial \Omega$ in $x$, i.e., $B_{x}\left(r_{i}\right) \subset \Omega\left(B_{x}\left(r_{e}\right) \cap \Omega=\varnothing\right)$. Define

$$
\delta_{i}=\inf \left\{r_{i}(x), x \in \partial \Omega\right\}, \quad \delta_{e}=\inf \left\{r_{e}(x), x \in \partial \Omega\right\}, \quad \delta=\min \left\{\delta_{i}, \delta_{e}\right\} .
$$

Since $\partial \Omega \in C^{2}$ we have $\delta>0$.

LEMmA 3.3. (G. Adler [1]) If $v \in C^{1}(\bar{\Omega}) \cap C^{2}(\bar{\Omega})$ is harmonic we have

$$
\max _{\Omega}|\nabla v| \leqslant C_{1} \frac{1}{\delta} \max _{\partial \Omega}|v(s)|+C_{2} \max _{\partial \Omega}\left|v^{\prime}(s)\right|+C_{3} \delta \max _{\partial \Omega}\left|v^{\prime \prime}(s)\right|
$$

where $s$ is the arc length along $\partial \Omega$ and $C_{1}, C_{2}, C_{3}$ are absolute constants.

Lemma 3.4. (D. Gilbarg and N. S. Trudinger [4], p. 37) Let $v \in C^{0}(\Omega) \cap C^{2}(\bar{\Omega})$ and $f$ bounded in $\Omega$. If $\Delta v=f$ we have

$$
d(x)|\nabla v(x)| \leqslant C\left(\sup _{\Omega}|v|+\sup _{\Omega} d^{2}(x)|f(x)|\right)
$$

where $d(x)=\operatorname{dist}\{x, \partial \Omega\}$.

The conclusions of the above lemmas remain true if $\Omega=s_{\varepsilon}$ and the function $v$ is 1 -periodic in $x_{1}$. This can be seen quite easily if we modify the proofs, reasoning in $o_{\varepsilon}$ and treating points like $\left(0, x_{2}\right),\left(1, x_{2}\right)$ as coincident interior points. In particular, we can take $\delta=\varepsilon / h_{M}$ in Lemma 3.3.

Define now in $s_{\varepsilon}$ the function $\psi_{0}\left(x_{1}, x_{2}\right)$ with the problem

$$
\begin{gathered}
\partial^{4} \psi_{0} / \partial x_{2}^{4}=0 \quad \text { in } s_{\varepsilon}, \\
\psi_{0}=0, \partial \psi_{0} / \partial x_{2}=1 \quad \text { on } x_{2}=0, \quad \psi_{0}=\varepsilon c_{0}, \partial \psi_{0} / \partial x_{2}=0 \quad \text { on } \quad x_{2}=\varepsilon h\left(x_{1}\right) .
\end{gathered}
$$

We find

$$
\psi_{0}\left(x_{1}, x_{2}\right)=\frac{\left(h-2 c_{0}\right) x_{2}^{3}}{\varepsilon^{2} h^{3}}+\frac{\left(3 c_{0}-2 h\right) x_{2}^{2}}{\varepsilon h^{2}}+x_{2} .
$$

We may regard $\psi_{0}$ as the stream function of the theory of lubrication. Indeed, let $v_{01}=\partial \psi_{0} / \partial x_{2} ;$ we have

$$
v_{01}=\frac{3\left(h-2 c_{0}\right) x_{2}^{2}}{\varepsilon^{2} h^{3}}+\frac{2\left(3 c_{0}-2 h\right) x_{2}}{\varepsilon h^{2}}+1
$$

and this is precisely the (nondimensional) $x_{1}$-component of the velocity given by the theory of lubrication. 
TheOREM 3.1. Suppose $c=c_{0} \varepsilon$ in Problem $2^{\prime}$. If $\mathbf{v}$ is the corresponding velocity and $k(\varepsilon)$ is given by

$$
p\left(x_{1}+1, x_{2}\right)-p\left(x_{1}, x_{2}\right)=k,
$$

then we have ${ }^{2}$

$$
\begin{gathered}
\left\|\nabla v_{1}\right\|_{L^{2}\left(o_{\varepsilon}\right)} \leqslant C \varepsilon^{-1 / 2}, \quad\left\|\nabla v_{2}\right\|_{L^{2}\left(o_{\varepsilon}\right)} \leqslant C, \\
\left\|v_{1}\right\|_{L^{p}\left(o_{\varepsilon}\right)} \leqslant C \varepsilon^{1 / p}, \quad\left\|v_{2}\right\|_{L^{p}\left(o_{\varepsilon}\right)} \leqslant C \varepsilon^{2 / p}, \quad 2<p<\infty, \\
|k(\varepsilon)| \leqslant C \varepsilon^{-2} .
\end{gathered}
$$

Proof. Define $\eta=\psi-\psi_{0}$ where $\psi$ is the solution of Problem $2^{\prime}$ and $\psi_{0}$ is given by (3.17). $n$ satisfies

$$
\begin{gathered}
\Delta^{2} \eta=f_{1} / \varepsilon^{2}+f_{2} / \varepsilon+f_{3}, \\
\eta=0, \quad d \eta / d n=0 \quad \text { on } \partial s_{\varepsilon},
\end{gathered}
$$

with $f_{1}, f_{2}$, and $f_{3}$ functions not depending on $\varepsilon$. Multiplying (3.22) by $\eta$ and integrating by parts over $o_{\varepsilon}$ we get, recalling that $\eta$ is 1-periodic in $x_{1}$,

$$
\left\|\eta_{x_{1} x_{1}}\right\|_{L^{2}\left(o_{\varepsilon}\right)}^{2}+2\left\|\eta_{x_{1} x_{2}}\right\|_{L^{2}\left(o_{\varepsilon}\right)}^{2}+\left\|\eta_{x_{2} x_{2}}\right\|_{L^{2}\left(o_{\varepsilon}\right)}^{2} \leqslant \frac{C}{\varepsilon^{2}}\|\eta\|_{L^{2}\left(o_{\varepsilon}\right)}
$$

By the Poincaré inequality we have

$$
\|\eta\|_{L^{2}\left(o_{\varepsilon}\right)} \leqslant C \varepsilon\left\|\eta_{x_{2}}\right\|_{L^{2}\left(o_{\varepsilon}\right)}, \quad\left\|\eta_{x_{2}}\right\|_{L^{2}\left(o_{\varepsilon}\right)} \leqslant C \varepsilon\left\|\eta_{x_{2} x_{2}}\right\|_{L^{2}\left(o_{\varepsilon}\right)} .
$$

Thus

$$
\|\eta\|_{L^{2}\left(o_{\varepsilon}\right)} \leqslant C \varepsilon^{2}\left\|\eta_{x_{2} x_{2}}\right\|_{L^{2}\left(o_{\varepsilon}\right)}
$$

Hence by (3.24),

$$
\left\|\eta_{x_{1} x_{1}}\right\|_{L^{2}\left(o_{\epsilon}\right)}^{2}+2\left\|\eta_{x_{1} x_{2}}\right\|_{L^{2}\left(o_{\epsilon}\right)}^{2}+\left\|\eta_{x_{2} x_{2}}\right\|_{L^{2}\left(o_{\epsilon}\right)}^{2} \leqslant C\left\|\eta_{x_{2} x_{2}}\right\|_{L^{2}\left(o_{\epsilon}\right)} .
$$

It follows that

$$
\begin{gathered}
\left\|\nabla \eta_{x_{1}}\right\|_{L^{2}}^{2}+\left\|\nabla \eta_{x_{2}}\right\|_{L^{2}}^{2} \leqslant C, \\
\left\|\eta_{x_{1}}\right\|_{L^{2}} \leqslant C \varepsilon, \quad\left\|\eta_{x_{2}}\right\|_{L^{2}} \leqslant C \varepsilon, \quad\|\eta\|_{L^{2}} \leqslant C \varepsilon^{2} .
\end{gathered}
$$

Moreover, we have

$$
\begin{aligned}
& \left\|\nabla v_{1}\right\|_{L^{2}}=\left\|\nabla \psi_{x_{2}}\right\|_{L^{2}} \leqslant\left\|\nabla \eta_{x_{2}}\right\|_{L^{2}}+\left\|\nabla \psi_{0_{x_{2}}}\right\|_{L^{2}}, \\
& \left\|\nabla v_{2}\right\|_{L^{2}}=\left\|\nabla \psi_{x_{1}}\right\|_{L^{2}} \leqslant\left\|\nabla \eta_{x_{1}}\right\|_{L^{2}}+\left\|\nabla \psi_{0_{x_{1}}}\right\|_{L^{2}},
\end{aligned}
$$

and by direct computation

$$
\left\|\nabla \psi_{0_{x_{2}}}\right\|_{L^{2}} \leqslant C \varepsilon^{-1 / 2}, \quad\left\|\nabla \psi_{0_{x_{1}}}\right\|_{L^{2}} \leqslant C \varepsilon^{1 / 2}
$$

Hence (3.19) holds true. By Lemma 3.1 and (3.25) we have

$$
\left\|\eta_{x_{1}}\right\|_{L^{p}} \leqslant C \varepsilon^{2 / p}, \quad\left\|\eta_{x_{2}}\right\|_{L^{p}} \leqslant C \varepsilon^{2 / p}
$$

\footnotetext{
${ }^{2}$ The various $C$ 's denote constants generally different, not depending on $\varepsilon$.
} 
Thus the estimates (3.20) follow if we note that

$$
\left\|\psi_{0_{x_{1}}}\right\|_{L^{p}} \leqslant C \varepsilon^{1+1 / p}, \quad\left\|\psi_{0_{x_{2}}}\right\|_{L^{p}} \leqslant C \varepsilon^{1 / p} \text {. }
$$

Finally we prove (3.21). Take $c=\varepsilon c_{0}$ and $c=2 \varepsilon c_{0}$ in Problem $2^{\prime}$ and let $\mathbf{v}$ and $\tilde{\mathbf{v}}$ be the corresponding velocities. Letting $\phi=\mathbf{v}-\tilde{\mathbf{v}}$ in the integral identity

$$
-[\mathbf{v}, \phi]=k(\varepsilon) \int_{0}^{\varepsilon h(0)} \phi_{1}\left(0, x_{2}\right) d x_{2}
$$

we obtain

$$
\varepsilon c_{0} k(\varepsilon)=-[\mathbf{v}, \tilde{\mathbf{v}}]+\|\mathbf{v}\|_{H}^{2} .
$$

Since, by (3.19),

$$
\|\mathbf{v}\|_{H} \leqslant C_{1} \varepsilon^{-1 / 2}, \quad\|\tilde{\mathbf{v}}\|_{H} \leqslant C_{2} \varepsilon^{-1 / 2}
$$

we get as a result

$$
c_{0}|k(\varepsilon)| \leqslant C \varepsilon^{-2} .
$$

Theorem 3.2. Suppose again $c=\varepsilon c_{0}$ in Problem $2^{\prime}$ and let $\mathbf{v}$ be the corresponding velocity. Then

$$
\|\mathbf{v}\|_{L^{\infty}} \leqslant C
$$

Proof. Let $\omega\left(x_{1}, x_{2}\right)$ be harmonic, 1-periodic in $x_{1}$ and such that $\omega=\psi$ on $\partial s_{\varepsilon}$. By Lemma 3.3, which is applicable in the present case, $|\nabla \omega|$ remains bounded as $\varepsilon \rightarrow 0$. Defining $\zeta=\psi-\omega$ we have

$$
\Delta^{2} \zeta=0 \text { in } s_{\varepsilon}, \quad \zeta=0 \text { on } \partial s_{\varepsilon} .
$$

Hence by Lemma 3.2,

$$
|\nabla \zeta| \leqslant \sqrt{56} \max _{\partial s_{\varepsilon}}|\partial \zeta / \partial n| \leqslant \sqrt{56}\left(\max _{\partial s_{\varepsilon}}|\partial \psi / \partial n|+\max _{\partial s_{\varepsilon}}|\partial \omega / \partial n|\right) .
$$

Since $|\partial \psi / \partial n| \leqslant 1$ and $|\nabla \omega|$ is bounded, we get $|\nabla \zeta| \leqslant C$. It follows that

$$
\|\nabla \psi\|_{p L^{\infty}} \leqslant C
$$

which implies (3.28).

In the following theorem we derive an interior pointwise estimate.

Theorem 3.3. Let $\psi$ be the solution of Problem $2^{\prime}$ when $c=\varepsilon_{0}$ and let $\psi_{0}$ be given by (3.17). Define $\eta=\psi-\psi_{0}$. Then

$$
d(x)|\nabla \eta(x)| \leqslant C \varepsilon^{2}
$$

where $d(x)=\operatorname{dist}\left\{x, \partial s_{\varepsilon}\right\}$.

Proof. The function $\eta$ satisfies (3.22) and (3.23). Let $\alpha$ be the solution of the problem

$$
\Delta \alpha=f_{2} / \varepsilon^{2}+f_{1} / \varepsilon+f_{0} \quad \text { in } \quad s_{\varepsilon}, \quad \alpha=0 \quad \text { on } \quad \partial s_{\varepsilon}, \quad \alpha\left(x_{1}+1, x_{2}\right)=\alpha\left(x_{1}, x_{2}\right)
$$

with $f_{i}$ as in (3.22). Define $\alpha_{B}=3 N x_{2}\left(\varepsilon h_{M}-x_{2}\right) /\left(2 \varepsilon^{2}\right), 0 \leqslant x_{2} \leqslant \varepsilon h_{M}$ where the constant $N$ is a pointwise bound for $\left|f_{i}\right|(i=1,2,3)$. By the maximum principle we have 
$|\alpha| \leqslant \alpha_{B}$ in $s_{\varepsilon}$ when $\varepsilon<1$. Hence

$$
|\alpha| \leqslant M, \quad M=3 N h_{M}^{2} / 8 .
$$

Suppose $\beta$ is defined by

$$
\Delta \beta=\alpha \quad \text { in } s_{\varepsilon}, \quad \beta=0 \quad \text { on } \quad \partial s_{\varepsilon}, \quad \beta\left(x_{1}+1, x_{2}\right)=\beta\left(x_{1}, x_{2}\right)
$$

and let $\beta_{B}=M x_{2}\left(\varepsilon h_{M}-x_{2}\right) / 2,0 \leqslant x_{2} \leqslant \varepsilon h_{M}$. Using again a comparison argument we find $|\beta| \leqslant \beta_{B}$; hence

$$
|\beta| \leqslant M h_{M}^{2} \varepsilon^{2} / 8 .
$$

Assume for the moment

$$
|\partial \beta / \partial n| \leqslant C \varepsilon .
$$

Since $d(x) \leqslant \varepsilon h_{M}$ and $\Delta \beta=\alpha$, we have by Lemma 3.4, recalling (3.31) and (3.32),

$$
d|\nabla \beta| \leqslant C_{1}\left(\sup _{s_{\varepsilon}}|\beta|+\sup _{s_{\varepsilon}} d^{2}|\alpha|\right) \leqslant C_{2} \varepsilon^{2} .
$$

Define $\gamma=\eta-\beta$. We have

$$
\Delta^{2} \gamma=0 \quad \text { in } s_{\varepsilon}, \quad \gamma=0 \text { on } \partial s_{\varepsilon} .
$$

Thus we get, by Lemma 3.2 and taking into account (3.23),

$$
|\nabla \gamma| \leqslant \sqrt{56} \max _{\partial s_{e}}|\partial \gamma / \partial n|=\sqrt{56} \max _{\partial s_{e}}|\partial \beta / \partial n| .
$$

On the other hand, by (3.33), (3.34), and (3.35), we obtain

$$
d|\nabla \eta| \leqslant d|\nabla \gamma|+d|\nabla \beta| \leqslant C \varepsilon^{2} .
$$

It remains to verify (3.33). Let $\delta$ be given by

$$
\Delta \delta=M \quad \text { in } \quad s_{\varepsilon}, \quad \delta=0 \quad \text { on } \quad \partial s_{\varepsilon}, \quad \delta\left(x_{1}+1, x_{2}\right)=\delta\left(x_{1}, x_{2}\right) .
$$

By (3.31) and the maximum principle we get $|\beta| \leqslant \delta$. On the other hand $\beta=\delta$ on $\partial s_{\varepsilon}$; consequently

$$
|\partial \beta / \partial n| \leqslant|\partial \delta / \partial n| \text { on } \partial s_{\varepsilon} .
$$

To estimate $|\partial \delta / \partial n|$ we transform (3.36) in a harmonic problem by letting $\xi=\delta-\beta_{B}$. We have

$$
\begin{gathered}
\Delta \xi=0 \quad \text { in } \quad s_{\varepsilon}, \\
\xi\left(x_{1}, 0\right)=0, \quad \xi\left(x_{1}, \varepsilon h\left(x_{2}\right)\right)=\varepsilon^{2} M h\left(x_{2}\right)\left(h\left(x_{2}\right)-h_{M}\right) / 2,
\end{gathered}
$$

and by Lemma $3.3,|\nabla \xi| \leqslant C_{3} \varepsilon$. Taking into account that $\left|\nabla \beta_{B}\right| \leqslant C_{4} \varepsilon$ it follows that $|\nabla \delta| \leqslant C_{5} \varepsilon$. Thus $|\partial \delta / \partial n| \leqslant C_{5} \varepsilon$ on $\partial s_{\varepsilon}$. This by (3.37) implies (3.33).

From (3.29) we deduce the estimates

$$
d(x)\left|v_{1}-v_{01}\right| \leqslant C \varepsilon^{2} .
$$

4. A perturbation technique. In this section we develop a perturbation scheme in $\varepsilon$ for Problem $2^{\prime}$. Since we prefer to work in a fixed domain we introduce the new variables

$$
y_{1}=x_{1}, \quad y_{2}=x_{2} / \varepsilon .
$$


In this way $s_{\varepsilon}$ and $o_{\varepsilon}$ become, respectively,

$$
\begin{aligned}
& s=\left\{\left(y_{1}, y_{2}\right) ;-\infty<y_{1}<\infty, 0<y_{2}<h\left(y_{1}\right)\right\}, \\
& o=\left\{\left(y_{1}, y_{2}\right) ; 0<y_{1}<1,0<y_{2}<h\left(y_{1}\right)\right\} .
\end{aligned}
$$

Hereafter we use for partial differentiation the notation

$$
D_{q_{1}, q_{2}}^{q} f=\partial^{q} f / \partial^{q_{1}} y_{1} \partial^{q_{2}} y_{2}, \quad q=q_{1}+q_{2} .
$$

Moreover, we put

$$
\nabla_{\varepsilon}=\left(\varepsilon D_{1,0}^{1}, D_{0,1}^{1}\right), \quad \Delta_{\varepsilon}=\varepsilon^{2} D_{2,0}^{2}+D_{0,2}^{2} .
$$

Problem $2^{\prime}$ can be rewritten as

Problem 3. To find $\phi$ such that

$$
\begin{gathered}
\quad \Delta_{\varepsilon}^{2} \phi=0 \quad \text { in } s, \\
\phi=0, \quad D_{0,1} \phi=\varepsilon \text { on } y_{2}=0, \\
\phi=\varepsilon c_{0}, \quad D_{0,1} \phi=0 \quad \text { on } y_{2}=h\left(y_{1}\right), \\
\phi \text { is 1-periodic in } y_{1},
\end{gathered}
$$

where of course $\phi\left(y_{1}, y_{2}\right)=\psi\left(y_{1}, \varepsilon y_{2}\right)$. Let $u_{i}\left(y_{1}, y_{2}\right)=v_{i}\left(y_{1}, \varepsilon y_{2}\right)$. Recalling the definition of stream function we have

$$
u_{1}=\frac{1}{\varepsilon} D_{0,1} \phi, \quad u_{2}=-D_{1,0} \phi
$$

Furthermore, for the pressure gradient, we get from (3.1) and (4.6)

$$
\begin{gathered}
D_{1,0} p=\frac{1}{\varepsilon} D_{2,1}^{3} \phi+\frac{1}{\varepsilon^{3}} D_{0,3}^{3} \phi, \\
\frac{1}{\varepsilon} D_{0,1} p=-D_{3,0}^{3} \phi-\frac{1}{\varepsilon^{2}} D_{1,2}^{3} \phi .
\end{gathered}
$$

Lemma 4.1. The solution $\phi_{\varepsilon}$ of Problem 3 is odd with respect to $\varepsilon$.

Proof. Because $\Delta_{\varepsilon}=\Delta_{-\varepsilon}$ and $\Delta_{\varepsilon}^{2}=\Delta_{-\varepsilon}^{2},-\phi_{-\varepsilon}$ satisfies Problem 3. Thus by the uniqueness of the solution we infer $\phi_{\varepsilon}=-\phi_{-\varepsilon}$.

By (4.6) $u_{1}$ is even with respect to $\varepsilon$ and $u_{2}$ is odd. Moreover, from (4.7) and (4.8), we see that $p$ is an even function of $\varepsilon$.

Set formally

$$
\phi=\phi^{(0)}+\varepsilon \phi^{(1)}+\varepsilon^{2} \phi^{(2)}+\varepsilon^{3} \phi^{(3)}+\cdots
$$

Substituting (4.9) into Problem 3 and equating like powers of $\varepsilon$ we obtain, in agreement with Lemma 4.1,

$$
\phi^{(2 i)}=0, \quad i=0,1, \ldots
$$


The coefficients of the odd powers of $\varepsilon$ satisfy the following hierarchy of boundary value problems:

$$
\begin{gathered}
D_{0,4}^{4} \phi^{(1)}=0 \text { in } s, \\
\phi^{(1)}=0, D_{0,1} \phi^{(1)}=1 \quad \text { on } y_{2}=0, \quad \phi^{(1)}=c_{0}, D_{0,1} \phi^{(1)}=0 \quad \text { on } y_{2}=h\left(h_{1}\right), \\
D_{0,4}^{4} \phi^{(3)}=-2 D_{2,2}^{4} \phi^{(1)} \quad \text { in } s, \\
\phi^{(3)}=0, D_{0,1} \phi^{(3)}=0 \quad \text { on } y_{2}=0 \text { and } y_{2}=h\left(y_{1}\right), \\
D_{0,4}^{4} \phi^{(2 j+1)}=-D_{4,0}^{4} \phi^{(2 j-3)}-2 D_{2,2}^{4} \phi^{(2 j-1)}, \quad j=2,3, \ldots, \\
\phi^{(2 j+1)}=0, D_{0,1} \phi^{(2 j+1)}=0 \quad \text { on } \quad y_{2}=0 \text { and } y_{2}=h\left(y_{1}\right)
\end{gathered}
$$

Problem (4.11)-(4.12) can be solved easily. We get (cf. (3.17))

$$
\phi^{(1)}=\left(h\left(y_{1}\right)-2 c_{0}\right) y_{2}^{3} / h^{3}\left(y_{1}\right)+\left(3 c_{0}-2 h\left(y_{1}\right)\right) y_{2}^{2} / h^{2}\left(y_{1}\right)+y_{2} .
$$

Recalling (4.10) we solve problems (4.13)-(4.14) and (4.15)-(4.16) recursively. We are led to the following formal expansion of $\phi$ in odd powers of $\varepsilon$ :

$$
\phi=\sum_{j=0}^{\infty} \varepsilon^{2 j+1} \phi^{(2 j+1)} .
$$

Define

$$
u_{1}^{(2 j)}=D_{0,1}^{1} \phi^{(2 j+1)}, \quad u_{2}^{(2 j+1)}=-D_{1,0}^{1} \phi^{(2 j+1)} .
$$

By (4.6) we have

$$
u_{1}=\sum_{j=0}^{\infty} \varepsilon^{2 j} u_{1}^{(2 j)}, \quad u_{2}=\sum_{j=0}^{\infty} \varepsilon^{2 j+1} u_{2}^{(2 j+1)} .
$$

Next we consider for $p$ an expansion of the form

$$
p=\sum_{k=-2}^{\infty} \varepsilon^{k} p^{(k)}
$$

Putting (4.21) into (4.7) and (4.8) we have:

$$
\begin{gathered}
D_{1,0} p^{(2 j+1)}=0, \quad D_{0,1} p^{(2 j+1)}=0, \quad j=-1,0,1, \ldots, \\
D_{1,0}^{1} p^{(-2)}=D_{0,3}^{3} \phi^{(1)}, \\
D_{1,0}^{1} p^{(2 j)}=D_{2,1}^{3} \phi^{(2 j+1)}+D_{0,3}^{3} \phi^{(2 j+3)}, \quad j=0,1,2, \ldots, \\
D_{0,1}^{1} p^{(-2)}=0, \quad D_{0,1}^{1} p^{(0)}=-D_{1,2}^{3} \phi^{(1)}, \\
D_{0,1}^{1} p^{(2 j)}=-D_{3,0}^{3} \phi^{(2 j-1)}-D_{1,2}^{3} \phi^{(2 j+1)} .
\end{gathered}
$$

By (4.22), $\nabla p^{(2 j+1)}=0$; therefore, apart from an inessential constant, we arrive at the formal expansion

$$
p=\sum_{j=-1}^{\infty} \varepsilon^{2 j} p^{(2 j)}
$$


Let us examine in greater detail the first term of $(4.20)_{1}$. From (4.17), we obtain (cf. (3.18)):

$$
u_{1}^{(0)}=3\left(h\left(y_{1}\right)-2 c_{0}\right) y_{2}^{2} / h^{3}\left(y_{1}\right)+2\left(3 c_{0}-2 h\left(y_{1}\right)\right) y_{2} / h^{2}\left(y_{1}\right)+1 .
$$

Furthermore, by $(4.25)_{1}, p^{(-2)}$ does not depend on $y_{2}$ and from (4.23) we have:

$$
d p^{(-2)} / d y_{1}=6\left(h\left(y_{1}\right)-2 c_{0}\right) / h^{3}\left(y_{1}\right) \text {. }
$$

We arrive, in conclusion, at the classical one-dimensional Reynolds equation:

$$
\frac{d}{d y_{1}}\left(h^{3} \frac{d p^{(-2)}}{d y_{1}}\right)=6 \frac{d h}{d y_{1}} \text {. }
$$

Moreover, from (4.28) and (4.29) we deduce:

$$
u_{1}^{(0)}=\frac{1}{2} \frac{d p^{(-2)}}{d y_{1}} y_{2}\left(y_{2}-h\left(y_{1}\right)\right)+\left(h\left(y_{1}\right)-y_{2}\right) / h\left(y_{1}\right) \text {. }
$$

Our goal is now to give a rigorous justification of the formal expansions (4.18), (4.20), and (4.21).

LEMMA 4.2. Define

$$
r(n)=\phi-\sum_{j=0}^{n} \varepsilon^{2 j+1} \phi^{(2 j+1)}
$$

We have

$$
\begin{gathered}
\left\|D_{1,0} r(n)\right\|_{L^{2}(o)} \leqslant C \varepsilon^{2 n+3}, \quad\left\|D_{0,1} r(n)\right\|_{L^{2}(o)} \leqslant C \varepsilon^{2 n+3}, \\
\left\|D_{2-i, i}^{2} r(n)\right\|_{L^{2}(o)} \leqslant C \varepsilon^{2 n+3}, \quad i=0,1,2 .
\end{gathered}
$$

Proof. By (4.2)

$$
\Delta_{\varepsilon}^{2} r(n)=-\sum_{j=0}^{n} \varepsilon^{2 j+1} \Delta_{\varepsilon}^{2} \phi^{(2 j+1)}
$$

We claim that

$$
\Delta_{\varepsilon}^{2} r(n)=\varepsilon^{2 n+3} F\left(y_{1}, y_{2} ; \varepsilon, n\right),
$$

where

$$
F\left(y_{1}, y_{2} ; \varepsilon, n\right)=-\left[D_{4,0}^{4} \phi^{(2 n-1)}+2 D_{2,2}^{4} \phi^{(2 n+1)}+\varepsilon^{2} D_{4,0}^{4} \phi^{(2 n+1)}\right] .
$$

The proof is by induction. For $n=1,(4.35)$ is true. Suppose (4.35) with $n$ arbitrary; then

$$
\begin{aligned}
\Delta_{\varepsilon}^{2} r(n+1)= & \Delta_{\varepsilon}^{2} r(n)-\varepsilon^{2 n+3} \Delta_{\varepsilon}^{2} \phi^{(2 n+3)} \\
= & -\varepsilon^{2 n+3}\left[D_{4,0}^{4} \phi^{(2 n-1)}+2 D_{2,2}^{4} \phi^{(2 n+1)}+D_{0,4}^{4} \phi^{(2 n+3)}\right] \\
& -\varepsilon^{2 n+5}\left[D_{4,0}^{4} \phi^{(2 n+1)}+2 D_{2,2}^{4} \phi^{(2 n+3)}+\varepsilon^{2} D_{4,0}^{4} \phi^{(2 n+3)}\right] .
\end{aligned}
$$

The first term in square brackets in the left-hand side vanishes by (4.15); thus (4.35) follows. We conclude that $F\left(y_{1}, y_{2} ; \varepsilon, n\right)$ is bounded pointwise by a constant not depending on $\varepsilon$. Because $r(n), r_{y_{1}}$, and $r_{y_{2}}$ vanish on $\partial s$, we have

$$
\left\|\Delta_{\varepsilon} r(n)\right\|_{L^{2}} \leqslant C \varepsilon^{2 n+3} \text {. }
$$

On the other hand,

$$
\left\|\Delta_{\varepsilon} r\right\|_{L^{2}}^{2}=\varepsilon^{4}\left\|D_{2,0}^{2} r\right\|_{L^{2}}^{2}+2 \varepsilon^{2}\left\|D_{1,1}^{2} r\right\|_{L^{2}}^{2}+\left\|D_{0,2}^{2} r\right\|_{L^{2}}^{2} .
$$


Hence

$$
\left\|D_{2-i, i}^{2} r(n)\right\|_{L^{2}} \leqslant C \varepsilon^{2 n+3-(2-i)}, \quad i=0,1,2 .
$$

Now (4.37) holds for every $n$ and

$$
r(n)=r(n+1)+\varepsilon^{2 n+3} \phi^{(2 n+3)} .
$$

Inequality (4.34) follows because

$$
\left\|D_{2-i, i}^{2} r(n)\right\|_{L^{2}} \leqslant\left\|D_{2-i, i}^{2} r(n+1)\right\|_{L^{2}}+C_{1} \varepsilon^{2 n+3} \leqslant C \varepsilon^{2 n+3} .
$$

On the other hand,

$$
\left\|D_{1,0}^{1} r(n)\right\|_{L^{2}} \leqslant C\left\|D_{1,1}^{2} r(n)\right\|_{L^{2}}, \quad\left\|D_{0,1}^{1} r(n)\right\|_{L^{2}} \leqslant C\left\|D_{0,2}^{2} r(n)\right\|_{L^{2}} ;
$$

thus (4.33) holds.

Let

$$
\rho\left(u_{1} ; n\right)=u_{1}-\sum_{j=0}^{n} \varepsilon^{2 j} u_{1}^{(2 j)}, \quad \rho\left(u_{2} ; n\right)=u_{2}-\sum_{j=0}^{n} \varepsilon^{2 j+1} u_{2}^{(2 j+1)}
$$

Since

$$
\rho\left(u_{1} ; n\right)=\frac{1}{\varepsilon} D_{0,1}^{1} r(n), \quad \rho\left(u_{2} ; n\right)=-D_{1,0}^{1} r(n),
$$

we have the following as an immediate consequence of Lemma 4.1.

THEOREM 4.1. The following estimates for $\rho\left(u_{1} ; n\right)$ and $\rho\left(u_{2} ; n\right)$ hold true:

$$
\begin{gathered}
\left\|\rho\left(u_{1} ; n\right)\right\|_{L^{2}} \leqslant C \varepsilon^{2 n+2}, \quad\left\|\rho\left(u_{2} ; n\right)\right\|_{L^{2}} \leqslant C \varepsilon^{2 n+3}, \\
\left\|D_{1-i, i}^{1} \rho\left(u_{1} ; n\right)\right\|_{L^{2}} \leqslant C \varepsilon^{2 n+2}, \quad\left\|D_{1-i, i}^{1} \rho\left(u_{2} ; n\right)\right\|_{L^{2}} \leqslant C \varepsilon^{2 n+3}, \quad i=0,1 .
\end{gathered}
$$

Remark 4.1. The classical theory of lubrication deals with $u_{1}^{(0)}$ and $u_{2}^{(1)}$. For these terms we have, in particular,

$$
\left\|u_{1}-u_{1}^{(0)}\right\|_{L^{2}} \leqslant C \varepsilon^{2}, \quad\left\|u_{2}-\varepsilon u_{2}^{(1)}\right\|_{L^{2}} \leqslant C \varepsilon^{3} .
$$

An interior estimate for the $L^{2}$-norms of higher-order derivatives of the stream function $\phi$ is given in the following.

Lemma 4.3. Let $\zeta$ be a $C^{\infty}$ function vanishing near $\partial s$ and 1-periodic in $y_{1}$. Define

$$
g=\Delta_{\varepsilon}^{N} r(n), \quad N=1,2, \ldots
$$

Then we have

$$
\begin{aligned}
& \left\|\nabla_{\varepsilon}(\zeta g)\right\|_{L^{2}} \leqslant C \varepsilon^{2 n+3}, \\
& \left\|\Delta_{\varepsilon}(\zeta g)\right\|_{L^{2}} \leqslant C \varepsilon^{2 n+3} .
\end{aligned}
$$

Proof. Let $N=1$. We have

$$
\begin{aligned}
\zeta g \Delta_{\varepsilon}(\zeta g) & =\zeta g\left(\zeta \Delta_{\varepsilon} g+2 \nabla_{\varepsilon} \zeta \cdot \nabla_{\varepsilon} g+g \Delta_{\varepsilon} \zeta\right) \\
& =\zeta^{2} g \Delta_{\varepsilon} g+\zeta g^{2} \Delta_{\varepsilon} \zeta+2 g \nabla_{\varepsilon} \zeta \cdot \nabla_{\varepsilon}(\zeta g)-2 g^{2}\left|\nabla_{\varepsilon} \zeta\right|^{2} .
\end{aligned}
$$


By (4.35) and (4.36) we get

$$
\|g\|_{L^{2}} \leqslant C \varepsilon^{2 n+3}, \quad\left\|\Delta_{\varepsilon} g\right\|_{L^{2}} \leqslant C \varepsilon^{2 n+3} .
$$

Integrating by parts in the left-hand side of (4.42) we get, taking into account (4.43),

$$
\left\|\nabla_{\varepsilon}(\zeta g)\right\|_{L^{2}}^{2} \leqslant{ }^{\cdot} C \varepsilon^{2 n+3}\left(\varepsilon^{2 n+3}+\left\|\nabla_{\varepsilon}(\zeta g)\right\|_{L^{2}}\right) .
$$

Hence (4.40) holds when $N=1$. In particular, we have from (4.40),

$$
\left\|\zeta D_{1,0}^{1} g\right\|_{L^{2}} \leqslant C \varepsilon^{2 n+2}, \quad\left\|\zeta D_{0,1}^{1} g\right\|_{L^{2}} \leqslant C \varepsilon^{2 n+3} .
$$

To prove (4.41) we note that:

$$
\begin{aligned}
\zeta g \Delta_{\varepsilon}^{2}(\zeta g) & =\zeta g\left\{\zeta \Delta_{\varepsilon}^{2} g+4 \nabla_{\varepsilon} \zeta \cdot \nabla_{\varepsilon}\left(\Delta_{\varepsilon} g\right)+2 \Delta_{\varepsilon} \zeta \Delta_{\varepsilon} g\right. \\
& \left.+\left[4\left(\varepsilon^{2} \nabla_{\varepsilon} D_{1,0} \zeta \cdot \nabla_{\varepsilon} D_{1,0} g+\nabla_{\varepsilon} D_{0,1} \zeta \cdot \nabla_{\varepsilon} D_{0,1} g\right)+4 \nabla_{\varepsilon} g \cdot \nabla_{\varepsilon}\left(\Delta_{\varepsilon} \zeta\right)\right]+g \Delta_{\varepsilon}^{2} \zeta\right\} .
\end{aligned}
$$

Recalling (4.35) we have

$$
\left\|\nabla_{\varepsilon}\left(\Delta_{\varepsilon} g\right)\right\|_{L^{2}} \leqslant C \varepsilon^{2 n+3}
$$

Hence by (4.43) and (4.46) the only terms in (4.45) which cannot be immediately estimated are those in square brackets. On the other hand, we can write

$$
\zeta g \nabla_{\varepsilon} g \cdot \nabla_{\varepsilon}\left(\Delta_{\varepsilon} \zeta\right)=\left(g \nabla_{\varepsilon}(\zeta g)-g^{2} \nabla_{\varepsilon} \zeta\right) \cdot \nabla_{\varepsilon}\left(\Delta_{\varepsilon} \zeta\right)
$$

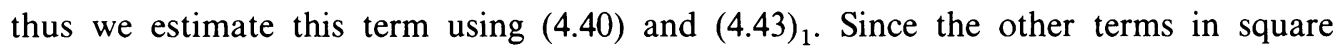
brackets in (4.45) can be similarly treated, we obtain (4.41) after integrating by parts in the left-hand side of (4.45). A suitable induction argument yields (4.40) and (4.41) when $N$ is arbitrary.

LEMmA 4.4. Let $\zeta$ be as in Lemma 4.3. We have, for $q \geqslant 2$ and $i=0,1, \ldots, q$,

$$
\left\|\zeta D_{q-i, i}^{q} r(n)\right\|_{L^{2}} \leqslant C \varepsilon^{2 n+3}
$$

Proof. We first prove by induction

$$
\left\|\zeta D_{q-i, i}^{q} r(n)\right\|_{L^{2}} \leqslant C \varepsilon^{2 n+3-(q-i)}
$$

when $2 \leqslant q \leqslant 2 n+3$ and $i=0,1, \ldots, q$. Inequality (4.48) holds when $q=2$ by (4.37). Suppose (4.48) true for $q=3, \ldots, 2 N$. We claim, for $i=0,1, \ldots, 2 N+1$,

$$
\left\|\zeta D_{2 N+1-i, i}^{2 N+1} r(n)\right\|_{L^{2}} \leqslant C \varepsilon^{2 n+3-(2 N+1-i)} .
$$

Let

$$
g=\Delta_{\varepsilon}^{N} r(n)=\sum_{k=0}^{N}\left(\begin{array}{l}
N \\
k
\end{array}\right) \varepsilon^{2(N-k)} D_{2(N-k), 2 k}^{2 N} r(n) .
$$

We want to rewrite (4.40) after collecting in the left-hand side all terms like

$$
\zeta D_{2 N+1-i, i}^{2 N+1} r(n) .
$$


We have

$$
\begin{aligned}
\left\|D_{1,0}^{1}(\zeta g)\right\|_{L^{2}}^{2}= & \int_{o} \sum_{k, h=0}^{N}\left[\left(\begin{array}{c}
N \\
k
\end{array}\right) \varepsilon^{2(N-k)} D_{1,0}^{1}\left(\zeta D_{2(N-k), 2 k}^{2 N} r(n)\right)\right] \\
& \times\left[\left(\begin{array}{c}
N \\
h
\end{array}\right) \varepsilon^{2(N-h)} D_{1,0}^{1}\left(\zeta D_{2(N-h), 2 h}^{2 N} r(n)\right)\right] d y_{1} d y_{2} .
\end{aligned}
$$

Let

$$
D_{1,0}^{1}\left(\zeta D_{2(N-k), 2 k}^{2 N} r(n)\right)=\zeta D_{2(N-k)+1,2 k}^{2 N+1} r(n)+\alpha_{k}
$$

where by the induction hypothesis

$$
\left\|\alpha_{k}\right\|_{L^{2}} \leqslant C \varepsilon^{2 n+3-2(N-k)}
$$

The diagonal terms in (4.52) can be estimated as follows:

$$
\left\|D_{1,0}^{1}\left(\zeta D_{2(N-k), 2 k}^{2 N} r(n)\right)\right\|_{L^{2}}^{2} \geqslant\left\|\zeta D_{2(N-k)+1,2 k}^{2 N+1} r(n)\right\|_{L^{2}}^{2}-2\left\|\alpha_{k}\right\|_{L^{2}}\left\|\zeta D_{2(N-k), 2 k}^{2 N+1} r(n)\right\|_{L^{2}} \text {. }
$$

For the off-diagonal terms in (4.52), we put

$$
D_{1,0}^{1}\left(\zeta D_{2(N-k), 2 k}^{2 N} r(n)\right)=D_{2(N-k)+1,2 k}^{2 N+1}(\zeta r(n))+\beta_{k}
$$

where

$$
\left\|\beta_{k}\right\|_{L^{2}} \leqslant C \varepsilon^{2 n+3-2(N-k)}
$$

Using (4.55) we have, with successive integrations by parts,

$$
\begin{aligned}
\int_{o} D_{1,0}^{1}\left(\zeta D_{2(N-k), 2 k}^{2 N} r(n)\right) \times D_{1,0}^{1}\left(\zeta D_{2(N-h), 2 h}^{2 N} r(n)\right) d y_{1} d y_{2} \\
=\int_{o}\left(D_{2 N+1-h-k, h+k}^{2 N+1}(\zeta r)\right)^{2} d y_{1} d y_{2}+\int_{o} \beta_{h} D_{2(N-k)+1,2 k}^{2 N+1}(\zeta r) d y_{1} d y_{2} \\
\quad+\int_{o} \beta_{k} D_{2(N-h)+1,2 h}^{2 N+1}(\zeta r) d y_{1} d y_{2}+\int_{o} \beta_{h} \beta_{k} d y_{1} d y_{2}
\end{aligned}
$$

To transform further the first term in the right-hand side of (4.57), we let

$$
D_{2 N+1-h-k, h+k}^{2 N+1}(\zeta r)=\zeta D_{2 N+1-h-k, h+k}^{2 N+1} r+\gamma
$$

where

$$
\|\gamma\|_{L^{2}} \leqslant C \varepsilon^{2 n+3-(2 N+1-h-k)} .
$$

Thus

$$
\left\|\zeta D_{2 N-h-k, h+k}^{2 N+1} r(n)\right\|_{L^{2}}^{2} \leqslant\left\|D_{2 N+1-h-k, h+k}^{2 N+1}(\zeta r)\right\|_{L^{2}}^{2}+2\|\gamma\|_{L^{2}}\left\|\zeta D_{2 N+1-h-k, h+k}^{2 N+1} r(n)\right\|_{L^{2}}
$$

Let us repeat the same calculations from (4.52) to (4.59) but starting with

$$
\left\|D_{0,1}^{1}(\zeta g)\right\|_{L^{2}}
$$

We finally obtain

$$
\sum_{i=0}^{2 N+1} \varepsilon^{2(2 N+1-i)}\left\|\zeta D_{2 N+1-i, i}^{2 N+1} r(n)\right\|_{L^{2}}^{2} \leqslant C \varepsilon^{2 n+3}\left(\varepsilon^{2 n+3}+\sum_{i=0}^{2 N+1} \varepsilon^{2 N+1-i}\left\|\zeta D_{2 N+1-i, i}^{2 N+1} r(n)\right\|_{L^{2}}\right) \text {. }
$$


Hence (4.49) follows. Let now (4.48) be true for $q=3, \ldots, 2 N+1$. We claim, for $i=0,1, \ldots, 2 N+2$,

$$
\left\|\zeta D_{2 N+2-i, i}^{2 N+2} r(n)\right\|_{L^{2}} \leqslant C \varepsilon^{2 n+3-(2 N+2-i)}
$$

Proceed now in a way similar to the first part of the proof, starting from (4.41). This inequality can be written as

$$
\varepsilon^{4}\left\|D_{2,0}^{2}(\zeta g)\right\|_{L^{2}}^{2}+2 \varepsilon^{2}\left\|D_{1,1}^{2}(\zeta g)\right\|_{L^{2}}^{2}+\left\|D_{0,2}^{2}(\zeta g)\right\|_{L^{2}}^{2} \leqslant C \varepsilon^{4 n+6}
$$

where $g$ is given again by (4.50). Our goal is to put in evidence and collect in the left-hand side of inequality (4.61) the $L^{2}$-norms of all terms like

$$
\zeta D_{2 N+2-i, i}^{2 N+2} r(n) \text {. }
$$

This can be done with estimates similar to those of the first part of the induction argument. As final result we obtain

$$
\sum_{i=0}^{2 N+2} \varepsilon^{2(2 N+2-i)}\left\|\zeta D_{2 N+2-i, i}^{2 N+2} r(n)\right\|_{L^{2}}^{2} \leqslant C \varepsilon^{2 n+3}\left(\varepsilon^{2 n+3}+\sum_{i=0}^{2 N+2} \varepsilon^{2 N+2-i}\left\|\zeta D_{2 N+2-i, i}^{2 N+2} r(n)\right\|_{L^{2}}\right) .
$$

Thus (4.60) follows. This completes the proof by induction of (4.48).

Take now $m$ such that $2 m>q$ and note that

$$
r(n)=r(n+m)+\sum_{j=n+1}^{n+m} \varepsilon^{2 j+1} \phi^{(2 j+1)} .
$$

Since (4.48) holds for every $n$ we have, for $i=0,1, \ldots, q$,

$$
\begin{aligned}
\left\|\zeta D_{q-i, i}^{q} r(n)\right\|_{L^{2}} & \leqslant\left\|\zeta D_{q-i, i}^{q} r(n+m)\right\|_{L^{2}}+\varepsilon^{2 n+3}\left\|\zeta \phi^{(2 n+3)}\right\|_{L^{2}} \\
& \leqslant C\left(\varepsilon^{2(n+m)+3-(q-i)}+\varepsilon^{2 n+3}\right) \\
& \leqslant C \varepsilon^{2 n+3} .
\end{aligned}
$$

This completes the proof of (4.47).

By the definitions of $\rho\left(u_{1}, n\right)$ and $\rho\left(u_{2}, n\right)$ we have, for $i=0,1, \ldots, m$,

$$
\zeta D_{m-i, i}^{m} \rho\left(u_{1}, n\right)=\frac{1}{\varepsilon} \zeta D_{m-i, i+1}^{m+1} r(n), \quad \zeta D_{m-i, i}^{m} \rho\left(u_{2}, n\right)=-\zeta D_{m-i+1, i}^{m+1} r(n) .
$$

Therefore, as an immediate consequence of Lemma 4.3, we obtain

THEOREM 4.2. For $m \geqslant 2$ and $i=0, \ldots, m$,

$$
\begin{gathered}
\left\|\zeta D_{m-i, i}^{m} \rho\left(u_{1}, n\right)\right\|_{L^{2}} \leqslant C \varepsilon^{2 n+2}, \\
\left\|\zeta D_{m-i, i}^{m} \rho\left(u_{2}, n\right)\right\|_{L^{2}} \leqslant C \varepsilon^{2 n+3} .
\end{gathered}
$$

Now we have, for $k=1,2$,

$$
\left\|\zeta D_{m-i, i}^{m} \rho\left(u_{k}, n\right)\right\|_{C^{0}(\bar{o})} \leqslant\left\|\zeta D_{m-i+1, i+1}^{m+2} \rho\left(u_{k}, n\right)\right\|_{L^{2}}
$$

Furthermore, the right-hand side in (4.63) and (4.64) does not depend on $m$. Thus we obtain, for $i=0,1, \ldots, m$,

$$
\left\|\zeta D_{m-i, i}^{m} \rho\left(u_{1}, n\right)\right\|_{C^{0}(\bar{o})} \leqslant C \varepsilon^{2 n+2}, \quad\left\|\zeta D_{m-i, i}^{m} \rho\left(u_{2}, n\right)\right\|_{C^{0}(\bar{o})} \leqslant C \varepsilon^{2 n+3} .
$$


To estimate the error associated with the pressure we define

$$
\tau(n)=p-\sum_{k=-1}^{n} \varepsilon^{2 k} p^{(2 k)} .
$$

By (4.7), (4.8), and (4.23)-(4.26), we have

$$
D_{1,0}^{1} \tau(n)=\frac{1}{\varepsilon^{3}} D_{0,3}^{3} r(n+1)+\frac{1}{\varepsilon} D_{2,1}^{3} r(n)
$$

and

$$
D_{0,1}^{1} \tau(n)=-\varepsilon D_{3,0}^{3} r(n-1)-\frac{1}{\varepsilon} D_{1,2}^{3} r(n) .
$$

Therefore we obtain, by (4.47) for $m \geqslant 1$ and $i=0,1, \ldots, m$,

$$
\left\|\zeta D_{m-i, i}^{m} \tau(n)\right\|_{L^{2}} \leqslant C \varepsilon^{2 n+2} .
$$

Acknowledgment. The author wishes to thank G. F. Capriz and G. Prodi for several helpful discussions and for their interest in this work.

\section{REFERENCES}

[1] G. Adler, Maggiorazione del gradiente delle funzioni armoniche mediante i loro valori al contorno, Mem. Acc. Naz. Lincei VI, 183-201 (1961)

[2] G. Cimatti, How the Reynolds equation is related to the Stokes equations, Appl. Math. and Opt. 10, 267-274 (1983)

[3] H. G. Elrod, A derivation of the basic equations for hydrodynamic lubrication with a fluid having constant properties, Quart. Appl. Math. 27, 349-359 (1960)

[4] D. Gilbarg and N. S. Trudinger, Elliptic partial differential equations of the second order, Springer, 1983

[5] A. Gross, Fluid film lubrication, John Wiley, 1980

[6] O. A. Ladyzhenskaya, The mathematical theory of viscous incompressible flow, Gordon and Breach, 1969

[7] C. Miranda, Formule di maggiorazione e teorema di esistenza per le funzioni biarmoniche in due variabili, Giorn. Mat. Battaglini 78, 97-118 (1948)

[8] O. Pincus and B. Sternlicht, Theory of hydrodynamic lubrication, McGraw-Hill, 1961

[9] O. Reynolds, On the theory of lubrication and its application to Mr. Beauchamp Tower's experiments including an experimental determination of the viscosity of olive oil, Phil. Trans. Roy. Soc. London 177, 157-234 (1886)

[10] G. H. Wannier, A contribution to the hydrodynamics of lubrication, Quart. Appl. Math. 8, 1-32 (1950)

[11] G. Bayada \& M. Chambat, The transition between the Stokes and the Reynolds equation: $A$ mathematical proof, Appl. Math. Optim. 14, 73-93 (1986) 